\section{Histone chaperone ASF1 cooperates with the Brahma chromatin-remodelling machinery}

\author{
Yuri M. Moshkin, ${ }^{1}$ Jennifer A. Armstrong, ${ }^{2}$ \\ Robert K. Maeda, ${ }^{1}$ John W. Tamkun, ${ }^{2}$ \\ Peter Verrijzer, ${ }^{3}$ James A. Kennison, ${ }^{4}$ and \\ Francois Karch ${ }^{1,5}$ \\ ${ }^{1}$ Department of Zoology and Animal Biology, University of \\ Geneva, 1211 Geneva 4, Switzerland; ${ }^{2}$ Department of \\ Molecular, Cell and Developmental Biology, University of \\ California, Santa Cruz, California 95064, USA; ${ }^{3}$ Department \\ of Molecular and Cell Biology, Medical Genetics Centre, \\ Centre for Biomedical Genetics, Leiden University Medical \\ Centre, 2300 RA Leiden, Netherlands; ${ }^{4}$ Laboratory of \\ Molecular Genetics, National Institute of Child Health and \\ Human Development, National Institutes of Health, \\ Bethesda, Maryland 20892, USA
}

De novo chromatin assembly into regularly spaced nucleosomal arrays is essential for eukaryotic genome maintenance and inheritance. The Anti-Silencing Function 1 protein (ASF1) has been shown to be a histone chaperone, participating in DNA-replication-coupled nucleosome assembly. We show that mutations in the Drosophila asf1 gene derepress silencing at heterochromatin and that the ASF1 protein has a cell cycle-specific nuclear and cytoplasmic localization. Furthermore, using both genetic and biochemical methods, we demonstrate that ASF1 interacts with the Brahma (SWI/SNF) chromatin-remodelling complex. These findings suggest that ASF1 plays a crucial role in both chromatin assembly and SWI/SNF-mediated chromatin remodelling.

Received March 20, 2002; revised version accepted August 16, 2002.

Assembly of newly synthesized DNA into chromatin requires both nucleosome assembly activities and ATPdependent chromatin-remodelling (Tyler and Kadonaga 1999; Philpott et al. 2000; Mello and Almouzni 2001). Nucleosome assembly is the process by which newly synthesized histones are loaded onto naked DNA. This function is performed primarily by histone chaperones like Chromatin Assembly Factor-1 (CAF-1) and Nucleosome Assembly Protein-1 (NAP-1; Tyler and Kadonaga 1999; Tyler et al. 1999; Munakata et al. 2000; Philpott et al. 2000; Mello and Almouzni 2001). However, nucleosome assembly factors alone are unable to efficiently produce long and regularly spaced nucleosomal arrays.

[Keywords: ASF1; Brahma; SWI/SNF complex; chromatin assembly; chromatin-remodelling]

${ }^{5}$ Corresponding author:

E-MAIL Francois.Karch@zoo.unige.ch; FAX 0041-22-702-6439.

Article and publication are at http://www.genesdev.org/cgi/doi/10.1101/ gad.231202.
To perform this function properly requires the recruitment of ATP-dependent chromatin-remodelling factors (Tyler and Kadonaga 1999; Mello and Almouzni 2001).

The asf 1 gene was originally identified in yeast by its ability, when overexpressed, to repress silencing at the $H M R$ and $H M L$ mating-type loci and at telomeres (Le et al. 1997). Interestingly, it has also been shown that lossof-function mutations in the yeast asf1 gene derepress transcription from silenced loci, when combined with mutations in the largest subunit of the yeast CAF-1 complex. Because of this, the role of ASF1 in silencing is thought to be in the assembly of silenced chromatin (Tyler et al. 1999; Sharp et al. 2001).

Recently, ASF1 has been shown to participate in the process of nucleosome assembly during DNA replication. Both biochemical and genetic studies have shown that ASF1 acts as a histone chaperone (Tyler et al. 1999, 2001; Munakata et al. 2000), which in concert with another histone chaperone, CAF-1, is thought to deposit histones $\mathrm{H} 3$ and $\mathrm{H} 4$ tetramers onto naked DNA. The assembly of nucleosome particles is completed by the addition of two dimers of histones $\mathrm{H} 2 \mathrm{~A}$ and $\mathrm{H} 2 \mathrm{~B}$, probably by the histone chaperone, NAP-1 (Luger et al. 1997; Tyler and Kadonaga 1999; Tyler et al. 1999; Philpott et al. 2000; Mello and Almouzni 2001).

Although most studies on ASF1 have focused on its role in nucleosome assembly, recent data have shown that the yeast ASF1 is required for the proper transcriptional repression and activation of the histone genes (Sutton et al. 2001). This role in transcription raises the possibility that ASF1 may play a role in chromatin remodelling, as well as nucleosome assembly. Here, we explore the function of ASF1 in chromatin dynamics and show that ASF1 is directly associated with the Brahma chromatin-remodelling machinery in flies.

\section{Results and Discussion}

Effect of asf1 mutation on heterochromatin-mediated silencing in flies

During an EMS saturation screen over the deficiency $D f(3 L) k t o 2$, which removes the $76 \mathrm{BD}$ region of the third chromosome, we identified two mutations in Drosophila asf1 gene $\left(a s f 1^{1}\right.$ and $\left.a s f 1^{2}\right)$.

The asf $1^{1}$ mutation deletes two nucleotides in the open reading frame (ORF) at base pair 380 relative to the "start" codon, creating a premature "stop" codon and resulting in the truncation of approximately half of the ASF1 protein (Fig. 1A). The protein synthesized from as $f 1^{1}$ mutant allele seems to be unstable. Although this protein still contains major epitopes recognized by our polyclonal anti-ASF1 antibodies, it cannot be detected in crude protein extracts from heterozygous as $f 1^{1}$ embryos (data not shown). Hemizygous as $\mathrm{f}^{1}$ mutants are embryonic or larval lethal; loss of maternal ASF1 function completely blocks oogenesis as revealed by as $f 1^{1}$ germline clones (data not shown).

The asf $1^{2}$ removes 24 nucleotides from the ORF of asf1 at base pair 54 after the "start" codon, resulting in an 8-amino-acid deletion in the protein (Fig. 1A). Because of the slight size difference between the mutant and wild-type proteins, we were unable to determine 


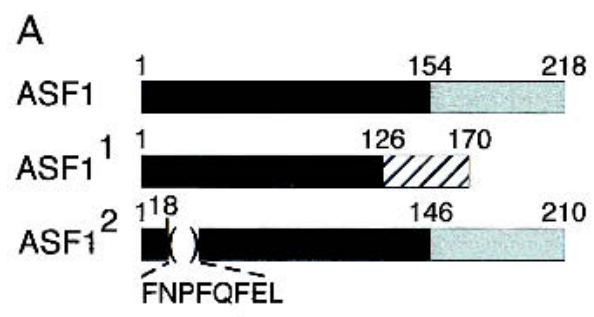

B

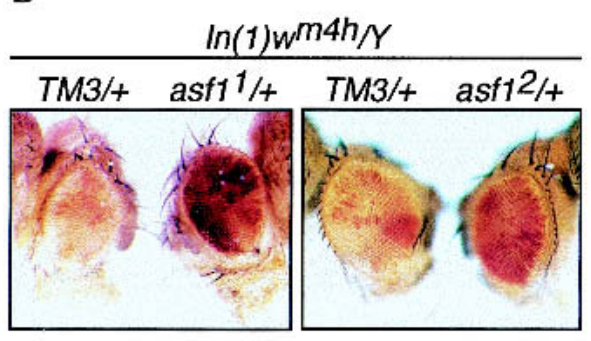

C

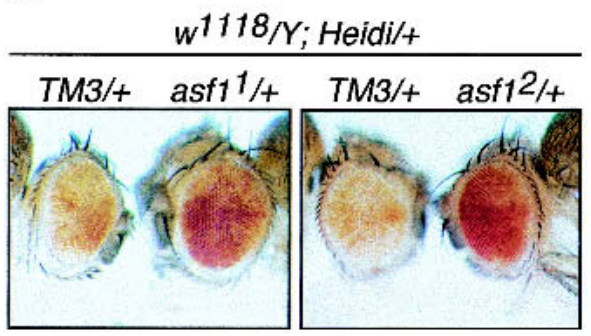

Figure 1. Suppression of position effect variegation $(\mathrm{PEV})$ by the asf1 mutations. (A) The N-terminal portion of the ASF1 protein (solid) is highly conserved among eukaryotes; the C-terminal portion (shaded) varies between species. The $a s f 1^{1}$ mutation creates a frame shift in the ASF1 protein after 126 amino acids, resulting in a truncated protein. The asf $1^{2}$ mutation deletes eight amino acids from the ASF1 protein. $(B)$ On the left, control males carrying the $\operatorname{In}(1) w^{m 4 h}$ inversion. The mottled eye color is the consequence of variegation of the white gene expression. Red spots are clones of cells expressing the white gene and white spots are clones of cells in which the white gene has been silenced by heterochromatin. On the right, males carrying $\operatorname{In}(1) w^{m 4 h}$ and heterozygous either for the as $f 1^{1}$ or as $f 1^{2}$ mutations. The eyes are almost red indicating that the white gene is derepressed, a characteristic of Suppressor of PEV mutations. $(C)$ Control males carrying the Heidi rearrangement show mosaic inactivation of the miniwhite reporter gene (left). Combining Heidi with the asf $1^{1}$ or as $f 1^{2}$ mutations result in the derepression of the miniwhite gene expression (right).

whether the $\mathrm{ASF} 1^{2}$ protein is present in heterozygous embryos. Histone-binding experiments, however, indicate that the mutated ASF1 protein produced by as $f 1^{2}$ allele shows markedly reduced binding to Drosophila histones $\mathrm{H} 3$ and $\mathrm{H} 4$ (data not shown).

Because ASF1 is involved in the assembly of silenced chromatin in yeast (Tyler et al. 1999; Sharp et al. 2001), we decided to test whether ASF1 is able to affect the silenced chromatin state at pericentric heterochromatin. We used the $\operatorname{In}(1) w^{m 4 h}$ and $\operatorname{In}(1) w^{m 4}$ mutant lines, which carry an inversion on the $\mathrm{X}$ chromosome juxtaposing the white gene to centromeric heterochromatin. This inversion leads to the classic position effect variegation (PEV) phenotype shown in Figure 1B. The cellautonomous inactivation of the white gene is thought to occur via the occasional spreading of the heterochromatic compaction of the DNA into the white gene. In flies heterozygous for the asf1 $1^{1}$ or $a s f 1^{2}$ mutations, we observe that the white gene expression is strongly derepressed in comparison to flies carrying two wild-type asf1 alleles (Fig. 1B).

To confirm the role of asf1 in heterochromatic silencing, we used the Heidi rearrangement as another PEV model. This chromosomal rearrangement juxtaposes a P-element transposon containing the miniwhite reporter gene next to centromeric heterochromatin on the second chromosome (Seum et al. 2000) and leads to the mosaic inactivation of the miniwhite expression in the eye (Fig. 1C). As with the $\operatorname{In}(1) W^{m 4}$ model, we find that both asf $1^{1}$ and $a s f 1^{2}$ mutations suppress heterochromatin-mediated inactivation of the miniwhite reporter gene (Fig. 1C).

The dominant suppression of PEV caused by mutations in the asf1 gene strongly suggests a function for ASF1 in the formation of silenced chromatin in Drosophila.

\section{Intracellular localization of ASF1 protein}

To gain more insight into ASF1 cellular function we raised and affinity-purified an antibody directed against the full-length ASF1 protein. This antibody recognizes a single band of $26 \mathrm{kD}$ in embryonic nuclear and crude extracts, which coincides with the predicted size of ASF1 and the size of bacterially expressed ASF1 protein (Fig. 2A).

We first looked at ASF1 localization on polytene chromosomes. ASF1 is strongly associated with multiple sites along the polytene chromosomes. Among them are many decondensed and transcriptionally active regions such as interbands and developmental puffs (Fig. 2B). Besides this, there is distinct staining of the chromocenter and the partially heterochromatic fourth chromosome, supporting the role of ASF1 in heterochromatin-mediated gene silencing (Fig. 2B,C). We also observe a particularly strong signal at the 39DE region (Fig. 2B,C). The $39 \mathrm{DE}$ region is the location of the histone gene cluster. Interestingly, ASF1 is known to be involved in the control of the histone genes expression in yeast, and the staining of the 39DE region may point to a similar role in flies (Sharp et al. 2001; Sutton et al. 2001).

Next, we analyzed the intracellular localization of ASF1 protein in the early Drosophila embryo. During the first hours of development, embryos undergo 13 cycles of nearly synchronous accelerated mitotic nuclear divisions, in which the G1 and G2 phases of the cell cycle are eliminated and cells only go through the $S$ and $M$ phases. Immunostaining with the anti-ASF1 antibody of these early embryos reveals that during S phase, ASF1 protein is primarily concentrated in the nucleus with only diffuse cytoplasmic staining (Fig. 3A). Because staining of the interphase cells of the salivary gland shows that nuclear ASF1 is associated with the chromosomes (Figs. $2 \mathrm{~B}, 3 \mathrm{C})$, it is likely that the early $\mathrm{S}$ phase embryonic staining is also chromosomal. Upon the commencement of mitosis, however, ASF1 nuclear staining fades and is not detected on the condensed chromatin (Fig. 3A,B).

\section{ASF1 cooperates in vivo with the Brahma chromatin-remodelling complex}

To further explore ASF1 function in the regulation of chromatin dynamics and to identify potential interacting partners, we created the eyeless-GAL4, UAS-Asf1 
A

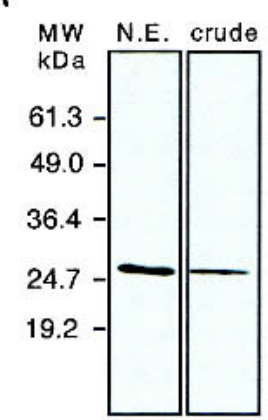

C
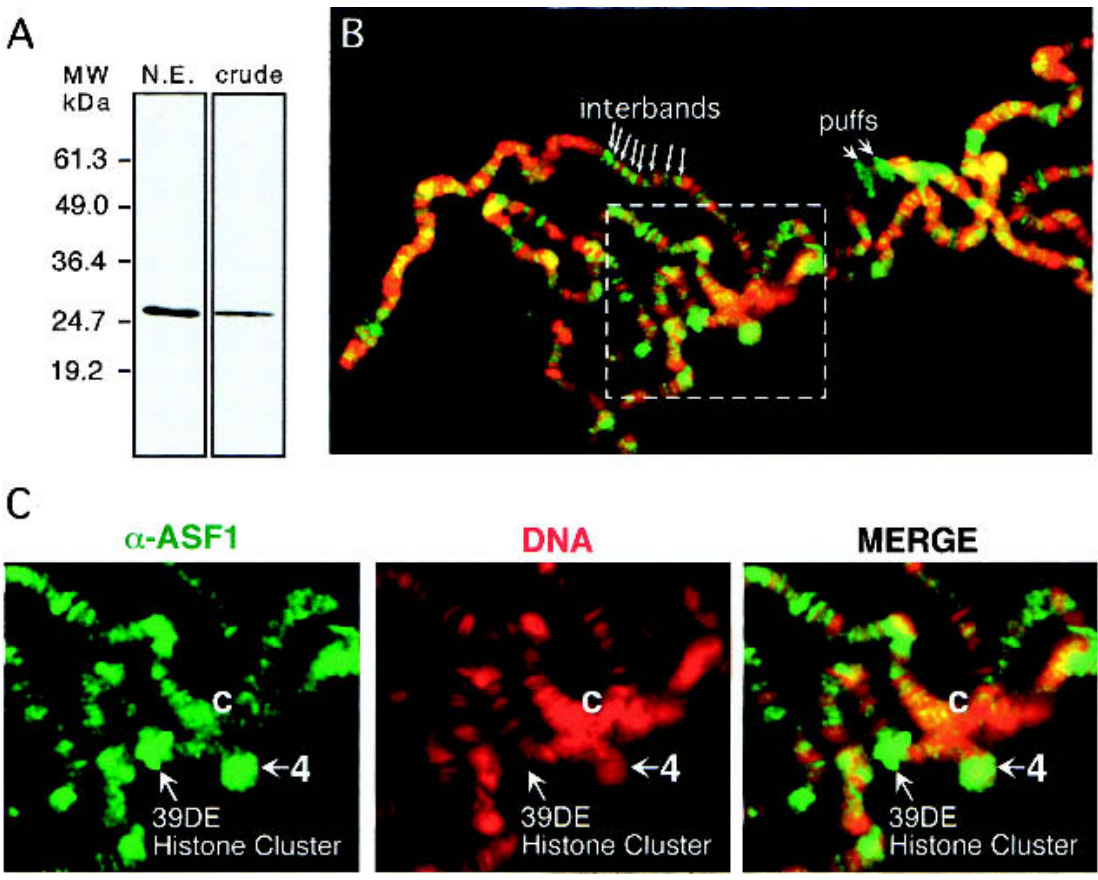

Figure 2. Localization of ASF1 protein on polytene chromosomes. (A) Immunoblot on embryonic nuclear (N.E.) and crude extracts with affinity-purified anti-ASF1 antibodies. $(B)$ Polytene chromosomes were stained for ASF1 protein with an anti-ASF1 antibody (green and for DNA with propidium iodide (red). ASF1 is localized to multiple sites on the chromosomal arms. Several interbands and puffs are indicated with arrows. $(C)$ A magnified view of the chromocenter, fourth chromosome, and 39DE region. ASF1 is strongly associated with the histone gene cluster, which is localized to the 39DE region of polytene chromosomes. ASF1 shows diffuse staining at the chromocenter (c) and also stains the fourth chromosome (4).

strain, which over-expresses asf1 cDNA in the eye. This strain has a rough-eye phenotype (Fig. 4A), which allows us to assay for genetic interactions between asf1 and genes known to be involved in the regulation of chromatin structure such as the Polycomb Group (PcG) and the Trithorax Group (TrxG) genes (Paro 1990; Simon et al. 1992; Kennison 1995; Mahmoudi and Verrijzer 2001). Among the tested mutations $\left(b_{\mathrm{rm}}{ }^{1}, \mathrm{brm}^{2}, \mathrm{mor}^{1}, \mathrm{osa}^{2}\right.$, $D f(3 R)$ red-P6, $\mathrm{ktO}^{1}, \operatorname{tara}^{\mathrm{L} 4}, \mathrm{AsX}^{\mathrm{Xf23}}, \mathrm{ph}^{410}, \mathrm{Pc}^{3}, \mathrm{Pcl}^{\mathrm{D5}}$, $\left.P_{S c^{1}}, E(z)^{S 4301}\right)$, we find that only mutations in the brahma (brm), moira (mor), and osa (osa) genes suppress the ASF1-mediated rough-eye phenotype (Fig. 4A). Interestingly, the proteins encoded by these genes are parts of the Brahma chromatin-remodelling complex (Papoulas et al. 1998; Collins et al. 1999; Kal et al. 2000).

To confirm the genetic interaction between ASF1 and the Brahma complex, we performed a reciprocal analysis. We used transgenic flies overexpressing a dominantnegative form of $\mathrm{brm}\left(\mathrm{brm}^{K 804 R}\right)$ in the eye (Elfring et al. 1998; Papoulas et al. 2001), which results in a rough-eye phenotype, similar to asf1 overexpression. In this assay, brm and mor mutations aggravate the effect of $b m^{K 804 R}$ over-expression, substantiating the dominant-negative nature of the brm $^{K 804 R}$ allele. Similarly, the $a s f 1^{1}$ mutation significantly enhances the rough-eye phenotype caused by overexpression of the dominantnegative brm ${ }^{K 804 R}$ allele (Fig. 4B). These two complementary genetic assays strongly suggest that ASF1 functions in vivo in the Brahma chromatin-remodelling pathway.
ASF1 is directly associated with the Brahma chromatin-remodelling complex in vitro

Because our genetic data show that ASF1 acts in the Brahma chromatin-remodelling pathway, we decided to test whether ASF1 directly interacts with the Brahma complex. Although the ASF1 protein is not found tightly associated with a highly purified Brahma complex (data not shown), we find that the BRM and its associated MOR proteins are coimmunoprecipitated with anti-ASF1 antibodies from embryonic nuclear extracts (Fig. 5A) suggesting that ASF1 does physically interact with the Brahma chromatin-remodelling complex. To test whether ASF1 can bind directly to the Brahma complex, we performed GST pull-down experiments using a bacterially expressed and purified ASF1-GST fusion protein and purified Brahma complex (Kal et al. 2000). Western blot analysis of pulled down material reveals that BRM, the ATPase subunit of the Brahma complex, is among the ASF1-interacting molecules (Fig. 5B) suggesting that ASF1 binds directly to the Brahma complex.

\section{Concluding remarks and perspectives}

We present evidence that Drosophila ASF1 plays a role in the formation of silenced chromatin similarly to its yeast counterpart (Tyler et al. 1999). Although the mechanism by which this is accomplished remains unclear, our data re-emphasize the importance of chromatin assembly factors in the formation of silenced chromatin. Because regularly spaced nucleosomal arrays are a landmark of silenced heterochromatin (Wallrath and Elgin 1995; Enomoto and Berman 1998), we believe that ASF1 contributes to silencing through its nucleosome assembly activity (Tyler et al. 1999). Therefore, the reduction of silencing in asf $1^{1}$ mutants may result from the disruption of the nucleosome array at heterochromatin. This interpretation is supported by the chromocentric localization of the ASF1 protein on polytene chromosomes (Fig. 2B,C).

We also found that ASF1 protein has a cell cycle-specific chromosomal and cytoplasmic localization reminiscent of another histone chaperone protein, NAP-1 (Ito et al. 1996). Ito et al. (1996) speculated that this localization pattern could reflect a role for NAP-1 in binding newly synthesized histones in the cytoplasm and delivering them to the sites of chromatin assembly and/or remodelling. We believe that ASF1 may play a similar role in histone shuttling to sites of chromatin assembly.

Furthermore, our data suggest a dualistic function for the histone chaperone ASF1 in both histones deposition during chromatin assembly and histones displacement during chromatin-remodelling (Fig. 5C). We find that ASF1 interacts genetically and biochemically with the Brahma chromatin-remodelling complex. The Dro- 


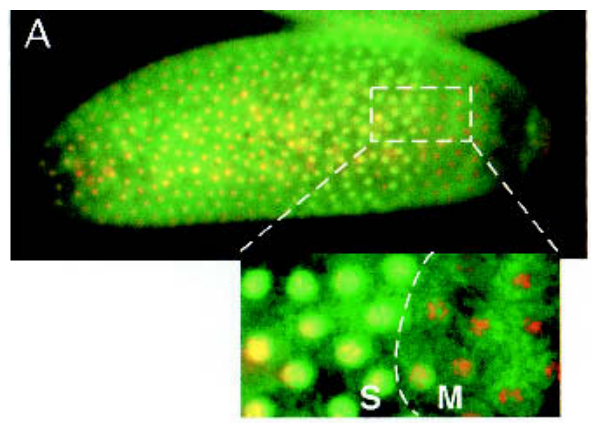

B

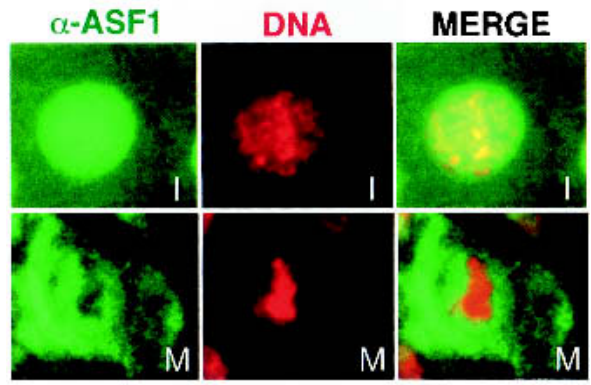

C

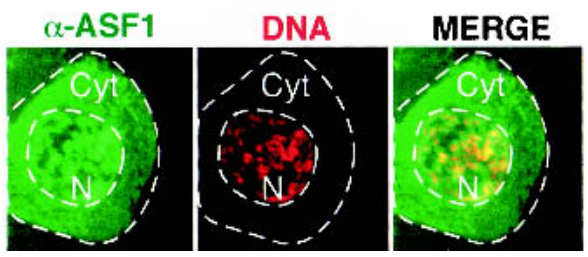

Figure 3. Intracellular localization of ASF1 protein. $(A)$ Localization of ASF1 protein in early embryos. Whole-mount early embryos were stained for ASF1 protein with an anti-ASF1 antibody (green) and for DNA with propidium iodide (red). Nuclear localization of ASF1 in S-phase nuclei (S) and its immediate dissociation from mitotic chromatin at prometaphase (M). (B) ASF1 protein localization during interphase and metaphase of cellularized embryos. $(C)$ Nuclear and cytoplasmic localization of ASF1 in salivary gland cells. ASF1 (green) colocalizes with chromatin (red). N, nucleus; Cyt, cytoplasm.

sophila Brahma complex is a member of the SWI/SNF ATP-utilizing chromatin-remodelling factors conserved in yeast, flies, and mammals (Papoulas et al. 1998; Tyler and Kadonaga 1999; Kal et al. 2000). As the Brahma complex participates in both the initiation and the repression of transcription (Kal et al. 2000), we believe that ASF1 may also function in transcriptional control. Although a direct role for ASF1 in transcription has not been firmly established, recent evidence supports this hypothesis. First, mutation of the yeast asf 1 gene results in the suppression of S-phase-specific histone genes activation (Sutton et al. 2001). Second, it was shown that ASF1 interacts with bromodomain-containing subunits of TFIID (Munakata et al. 2000; Chimura et al. 2002).

The association of ASF1 with the chromatin-remodelling machinery raises several intriguing possibilities for ASF1 function in chromatin-remodelling. As a histone chaperone, ASF1 could facilitate chromatin-remodelling by attenuating the strong electrostatic histone-DNA contacts, in effect, lubricating the chromatin for remodelling factors. Recently, it has been shown that the disruption of a single histone-DNA contact by a mutation in the SIN domain of histone $\mathrm{H} 4$ results in an increased rate of remodelling by the yeast SWI/SNF complex (Horn et al. 2002). In a similar fashion, ASF1 may weaken the contacts of histones $\mathrm{H} 3$ and $\mathrm{H} 4$ with DNA creating an altered nucleosome structure favorable for translocation by remodelling factors.

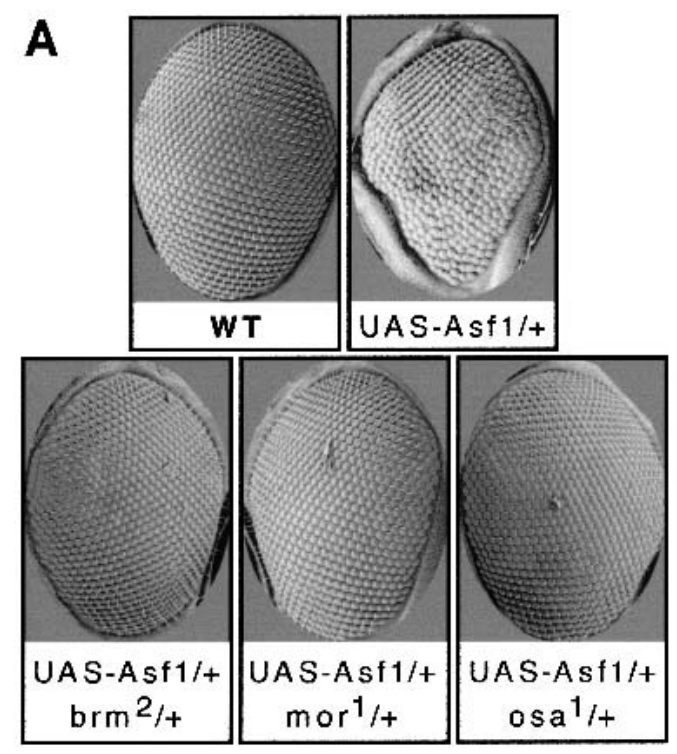

B

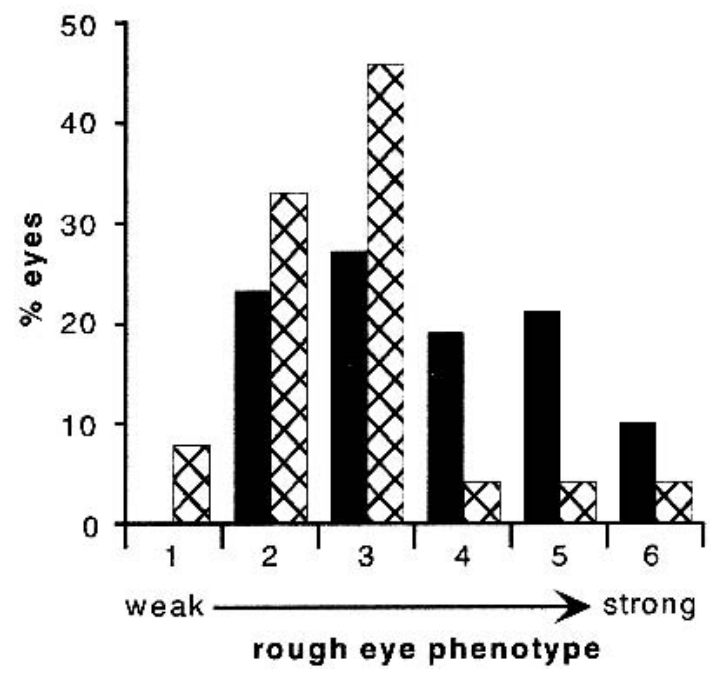

Figure 4. Genetic interaction between ASF1 and the Brahma chromatin-remodelling complex. (A) Flies carrying the UAS-Asf1 transgene recombined with the eyeless-GAL4 driver on the second chromosome. Heterozygous recombinant eyeless-GAL4, UAS-Asf1/+ flies $(U A S-A s f 1 /+)$ display a distinct rough-eye phenotype in comparison with the wild-type Oregon R strain (WT). The mutant alleles of the brm, mor, and osa TrxG genes suppress the UAS-Asf1-mediated rough-eye phenotype. (B) Enhancement of the brm ${ }^{K 804 R}$ dominant-negative rough-eye phenotype by the $a s f 1^{1}$ mutant allele. Hatched bars, eyeless-GAL4, UAS-brm ${ }^{K 804 R / T M 3}$, Sb, Ser; solid bars, eyeless-GAL4, UAS-brm ${ }^{K 804 R} / a^{a s f 1^{1}}$. Flies were sorted in six categories according to the severity of the eye phenotype. (1) Normal eye; (2) eye $\leqq 50 \%$ rough; (3) eye $>50 \%$ rough; (4) eye rough and reduced in size by $\leqq 50 \%$; $(5)$ eye rough and reduced in size by $>50 \%$; (6) no ommatedia. $(P<0.025$; Kolmogorov-Smirnov two-sample test). 
A

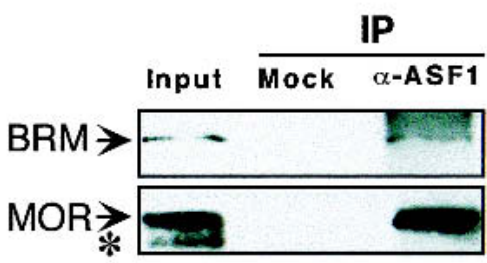

B

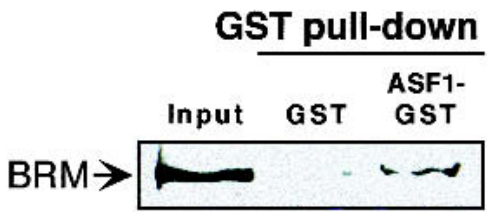

C

Chromatin assembly

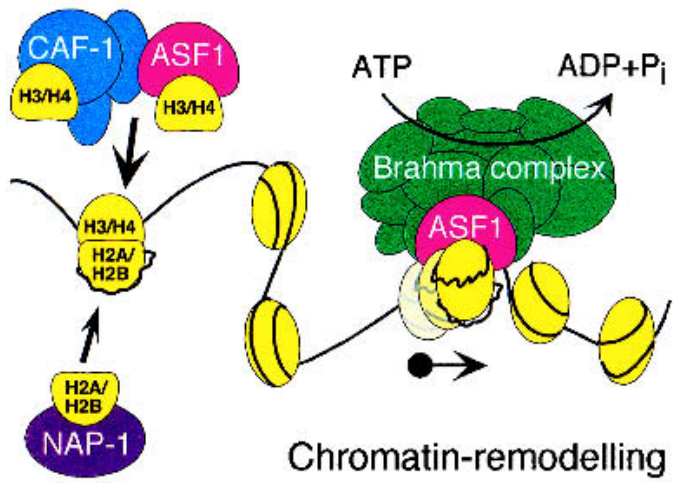

Figure 5. In vitro interaction of ASF1 with the Brahma chromatin-remodelling complex. $(A)$ The BRM and MOR proteins are immunoprecipitated from embryonic nuclear extracts with the anti-ASF1 antibody ( $\alpha$-ASF1) but not with rabbit pre-immune serum (Mock) as revealed by immunoblots using antiBRM or anti-MOR antibodies. Input mixture $(5 \%)$ was loaded as an input control (Input). Asterisk indicates an additional band of lower molecular weight recognized by anti-MOR antibody from embryonic nuclear extract, which is not precipitated by antiASF1 antibody $(\alpha$-ASF1). (B) GST pull-down assays with the Brahma complex. BRM protein associates with the ASF1-GSTfusion protein but not with the GST protein alone, as detected by immunoblots using anti-BRM antibodies. Input mixture $(5 \%)$ was loaded as an input control (Input). (C) Function of histone chaperone ASF1 in chromatin assembly and remodelling. 1) ASF1 functions synergistically with CAF-1 in deposition of histones $\mathrm{H} 3$ and $\mathrm{H} 4$ onto newly replicated DNA. Histones $\mathrm{H} 2 \mathrm{~A}$ and $\mathrm{H} 2 \mathrm{~B}$ are deposited by the histone chaperone NAP-1. 2) Histone chaperone ASF1 interacts directly with the Brahma complex suggesting the function for ASF1 in ATP-dependent chromatin-remodelling.

On the other hand, ASF1 could function in targeting chromatin-remodelling factors to the sites of newly assembled chromatin. As assembly of long and regularly spaced nucleosome arrays cannot be achieved by histone chaperones alone (Tyler and Kadonaga 1999; Philpott et al. 2000; Mello and Almouzni 2001) and some chromatin assembly complexes contain ATP-dependent nucleosome spacing activity (Ito et al. 1999; Bozhenok et al. 2002), an interaction between ASF1 and chromatin-re- modelling factors could indicate a mechanism by which functional chromatin is assembled after DNA replication.

\section{Materials and methods}

Fly stocks, P-element construction, and genetics Chromosomes and mutations are described in Flybase (http://flybase.bio. indiana.edu). All crosses were done at $25^{\circ} \mathrm{C}$ on a standard medium.

asf1 was mapped to 3-46.2 on the meiotic map, between the markers $c p$ (3-45.3) and in (3-46.75). Approximately 1000 progeny from mothers heterozygous for $c p^{1}, \mathrm{in}^{1}$, and $a s f 1^{1}$ were scored. Nine recombinants between $c p^{1}$ and $a s f 1^{1}$ and five recombinants between $a s f 1^{1}$ and $i n^{1}$ were recovered and tested for dominant suppression of $\operatorname{In}(1) W^{m 4}$ variegation. None of the recombinants separated the suppression of $\operatorname{In}(1) W^{m 4}$ from the as $f 1^{1}$ mutation.

To create the UAS-Asf1 transgenic lines, full-length cDNA of asf1 was inserted into the P-element vector, pUAST, downstream of the yeast Upstream Activating Sequences (UAS; Brand and Perrimon 1993) and transformed into a $W^{-}$strain. To produce the eyeless-GAL4, UAS-Asf1 strain, we recombined a $U A S-A s f 1$ P-element insertion with the eyelessGAL4 driver on the second chromosome.

\section{Anti-ASF1 antibody production and purification}

For producing ASF1-specific antibodies, a bacterially expressed ASF1GST fusion protein was injected into rabbits (Elevage Scientifique Des Dombes). Crude antiserum was purified by affinity chromatography using the ASF1-GST protein coupled to Affi-Gel 15 resin (Bio-Rad). All immunologic procedures were performed with affinity-purified antiASF1 antibodies. Immunoblotting experiments were performed with either the ECL detection system following manufacturer's instructions (Amersham) or the Alkaline Phosphatase developing system (Roche).

\section{Immunostaining techniques}

Immunostainings of the early embryos (1-2 h) were conducted using a 1:1000 diluted primary anti-ASF1 antibody and a DTAF-conjugated secondary antibody (Jackson ImmunoResearch Laboratories) diluted 1:200 (Cleard et al. 1997). DNA was counterstained with propidium iodide after RNase treatment. Immunostaining of polytene chromosomes from salivary glands was done using a 1:100 dilution of anti-ASF1 antibody (Platero et al. 1995).

Coimmunoprecipitation experiments with embryonic nuclear extract For coimmunoprecipitation experiments antibodies were coupled to Protein-A beads (Pharmacia Biotech) and incubated with Drosophila embryonic nuclear extracts for $2 \mathrm{~h}$ at $4^{\circ} \mathrm{C}$ in precipitation buffer $(12 \mathrm{mM}$ Hepes$\mathrm{KOH}$ at $\mathrm{pH} 7.8 ; 4 \mathrm{mM}$ Tris- $\mathrm{HCl}$ at $\mathrm{pH} 7.8 ; 60 \mathrm{mM} \mathrm{KCl} ; 5 \mathrm{mM} \mathrm{MgCl}_{2} ; 0.1$ mM EDTA; 0.5 mM DTT; $0.1 \%$ NP-40; $10 \%$ glycerol) in the presence of protease inhibitors (Roche). Affinity resins were subsequently washed three times with 10 bed volumes of the precipitation buffer without glycerol and proteins were separated by SDS-PAGE on $8 \%$ gels. The BRM and MOR proteins were detected by immunoblotting with anti-BRM and anti-MOR antibodies (Kal et al. 2000).

GST pull-down experiments with Drosophila core histones and purified Brahma complex

The ASF1-GST and mutated ASF1 ${ }^{1-G S T}$ and ASF1 ${ }^{2}$-GST fusion proteins were produced in bacteria. GST pull-down experiments with Drosophila core histones were performed as described (Katsani et al. 2001).

The Brahma chromatin-remodelling complex was purified from embryonic nuclear extract by chromatography on POROS-Heparin, Sephacryl S-300, and Bioscale Q10 columns (Kal et al. 2000). GST pull-down experiments with the Brahma complex were performed for $2 \mathrm{~h}$ at $4{ }^{\circ} \mathrm{C}$ in binding buffer $(20 \mathrm{mM}$ Hepes- $\mathrm{KOH}$ at $\mathrm{pH} 7.6 ; 60 \mathrm{mM} \mathrm{KCl} ; 2.5 \mathrm{mM}$ $\mathrm{MgCl}_{2} ; 10 \%$ glycerol; $0.05 \%$ NP-40; 1 mM DTT) with ASF1-GSTor GST (negative control) coupled to the Glutathione Sepharose 4B beads (Pharmacia Biotech). Beads were subsequently washed three times with 10 bed volumes of the binding buffer without glycerol and proteins were separated by SDS-PAGE on $8 \%$ gels. The BRM protein was detected by immunoblotting with an anti-BRM antibody (Kal et al. 2000).

\section{Acknowledgments}

We are indebted to E. Nigg and $\mathrm{H}$. Sillje who directed us to ASF1. We thank A. Kal, K. Katsani, G. Chalkley, O. Papoulas, M. Pilyugin for their 
help with biochemical experiments; Dr J. Wuest for scanning EM photographs; and A. Spierer for her help in making UAS-Asf1 transgenic lines. We also thank E. Favre and G. Faustino for excellent technical assistance. Finally, we especially thank Pierre Spierer for support. This work was supported by grants from the Swiss National Foundation and by the state of Geneva as well as by a grant from the National Institutes of Health to J.W.T. (GM49883). J.A.A. was supported by the Damon Runyon Cancer Research Foundation Fellowship (DRG-1556).

The publication costs of this article were defrayed in part by payment of page charges. This article must therefore be hereby marked "advertisement" in accordance with 18 USC section 1734 solely to indicate this fact.

\section{References}

Bozhenok, L., Wade, P.A., and Varga-Weisz, P. 2002. WSTF-ISWI chromatin remodeling complex targets heterochromatic replication foci. EMBO I 21: 2231-2241.

Brand, A.H. and Perrimon, N. 1993. Targeted gene expression as a means of altering cell fates and generating dominant phenotypes. Development 118: 401-415.

Chimura, T., Kuzuhara, T., and Horikoshi, M. 2002. Identification and characterization of CIA/ASF1 as an interactor of bromodomains associated with TFIID. Proc. Natl. Acad. Sci. 99: 9334-9339.

Cleard, F., Delattre, M., and Spierer, P. 1997. SU(VAR)3-7, a Drosophila heterochromatin-associated protein and companion of HP1 in the genomic silencing of position-effect variegation. EMBO J. 16: 52805288.

Collins, R.T., Furukawa, T., Tanese, N., and Treisman, J.E. 1999. Osa associates with the Brahma chromatin remodeling complex and promotes the activation of some target genes. EMBO J. 18: 7029-7040.

Elfring, L.K., Daniel, C., Papoulas, O., Deuring, R., Sarte, M., Moseley, S., Beek, S.J., Waldrip, W.R., Daubresse, G., DePace, A., et al. 1998. Genetic analysis of brahma: The Drosophila homolog of the yeast chromatin remodeling factor SWI2/SNF2. Genetics 148: 251-265.

Enomoto, S. and Berman, J. 1998. Chromatin assembly factor I contributes to the maintenance, but not the re-establishment, of silencing at the yeast silent mating loci. Genes \& Dev. 12: 219-232.

Horn, P.J., Crowley, K.A., Carruthers, L.M., Hansen, J.C., and Peterson, C.L. 2002. The SIN domain of the histone octamer is essential for intramolecular folding of nucleosomal arrays. Nat. Struct. Biol. 11: 11.

Ito, T., Bulger, M., Kobayashi, R., and Kadonaga, J.T. 1996. Drosophila NAP-1 is a core histone chaperone that functions in ATP-facilitated assembly of regularly spaced nucleosomal arrays. Mol. Cell. Biol. 16: 3112-3124.

Ito, T., Levenstein, M.E., Fyodorov, D.V., Kutach, A.K., Kobayashi, R., and Kadonaga, J.T. 1999. ACF consists of two subunits, Acf1 and ISWI, that function cooperatively in the ATP-dependent catalysis of chromatin assembly. Genes \& Dev. 13: 1529-1539.

Kal, A.J., Mahmoudi, T., Zak, N.B., and Verrijzer, C.P. 2000. The Drosophila brahma complex is an essential coactivator for the trithorax group protein zeste. Genes \& Dev. 14: 1058-1071.

Katsani, K.R., Arredondo, J.J., Kal, A.J., and Verrijzer, C.P. 2001. A homeotic mutation in the trithorax SET domain impedes histone binding. Genes \& Dev. 15: 2197-2202.

Kennison, J.A. 1995. The Polycomb and trithorax group proteins of Drosophila: Trans-regulators of homeotic gene function. Annu. Rev. Genet. 29: 289-303.

Le, S., Davis, C., Konopka, J.B., and Sternglanz, R. 1997. Two new Sphase-specific genes from Saccharomyces cerevisiae. Yeast 13: 10291042.

Luger, K., Mader, A.W., Richmond, R.K., Sargent, D.F., and Richmond, T.J. 1997. Crystal structure of the nucleosome core particle at $2.8 \AA$ resolution. Nature 389: 251-260.

Mahmoudi, T. and Verrijzer, C.P. 2001. Chromatin silencing and activation by Polycomb and trithorax group proteins. Oncogene 20: 3055 3066.

Mello, J.A. and Almouzni, G. 2001. The ins and outs of nucleosome assembly. Curr. Opin. Genet. Dev. 11: 136-141.

Munakata, T., Adachi, N., Yokoyama, N., Kuzuhara, T., and Horikoshi, M. 2000. A human homologue of yeast anti-silencing factor has histone chaperone activity. Genes Cells 5: 221-233.
Papoulas, O., Beek, S.J., Moseley, S.L., McCallum, C.M., Sarte, M., Shearn, A., and Tamkun, J.W. 1998. The Drosophila trithorax group proteins BRM, ASH1 and ASH2 are subunits of distinct protein complexes. Development 125: 3955-3966.

Papoulas, O., Daubresse, G., Armstrong, J.A., Jin, J., Scott, M.P., and Tamkun, J.W. 2001. The HMG-domain protein BAP111 is important for the function of the BRM chromatin-remodeling complex in vivo. Proc. Natl. Acad. Sci. 98: 5728-5733.

Paro, R. 1990. Imprinting a determined state into the chromatin of Drosophila. Trends Genet. 6: 416-421.

Philpott, A., Krude, T., and Laskey, R.A. 2000. Nuclear chaperones. Semin. Cell. Dev. Biol. 11: 7-14.

Platero, J.S., Hartnett, T., and Eissenberg, J.C. 1995. Functional analysis of the chromo domain of HP1. EMBO J. 14: 3977-3986.

Seum, C., Spierer, A., Delattre, M., Pauli, D., and Spierer, P. 2000. A GAL4-HP1 fusion protein targeted near heterochromatin promotes gene silencing. Chromosoma 109: 453-459.

Sharp, J.A., Fouts, E.T., Krawitz, D.C., and Kaufman, P.D. 2001. Yeast histone deposition protein Asflp requires Hir proteins and PCNA for heterochromatic silencing. Curr. Biol. 11: 463-473.

Simon, J., Chiang, A., and Bender, W. 1992. Ten different Polycomb group genes are required for spatial control of the abdA and AbdB homeotic products. Development 114: 493-505.

Sutton, A., Bucaria, J., Osley, M.A., and Sternglanz, R. 2001. Yeast ASF1 protein is required for cell cycle regulation of histone gene transcription. Genetics 158: 587-596.

Tyler, J.K. and Kadonaga, J.T. 1999. The "dark side" of chromatin remodeling: Repressive effects on transcription. Cell 99: 443-446.

Tyler, J.K., Adams, C.R., Chen, S.R., Kobayashi, R.,Kamakaka, R.T., and Kadonaga, J.T. 1999. The RCAF complex mediates chromatin assembly during DNA replication and repair. Nature 402: 555-560.

Tyler, J.K., Collins, K.A., Prasad-Sinha, J., Amiott, E., Bulger, M., Harte, P.J., Kobayashi, R., and Kadonaga, J.T. 2001. Interaction between the Drosophila CAF-1 and ASF1 chromatin assembly factors. Mol. Cell. Biol. 21: 6574-6584.

Wallrath, L.L. and Elgin, S.C. 1995. Position effect variegation in Drosophila is associated with an altered chromatin structure. Genes \& Dev. 9: 1263-1277. 


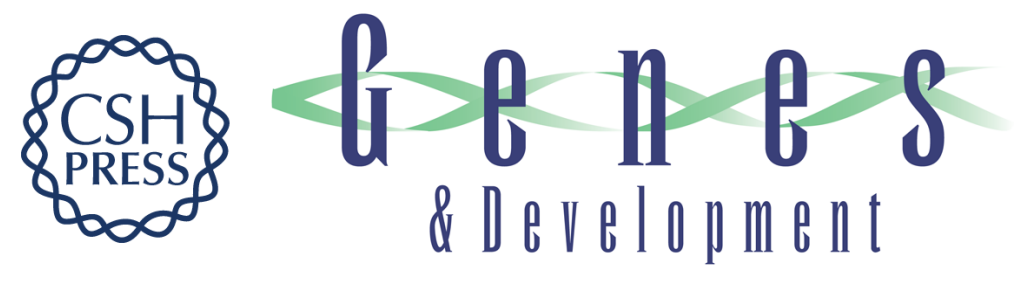

\section{Histone chaperone ASF1 cooperates with the Brahma chromatin-remodelling machinery}

Yuri M. Moshkin, Jennifer A. Armstrong, Robert K. Maeda, et al.

Genes Dev. 2002, 16:

Access the most recent version at doi:10.1101/gad.231202

References This article cites 31 articles, 17 of which can be accessed free at: http://genesdev.cshlp.org/content/16/20/2621.full.html\#ref-list-1

License

Email Alerting

Receive free email alerts when new articles cite this article - sign up in the box at the top Service right corner of the article or click here.

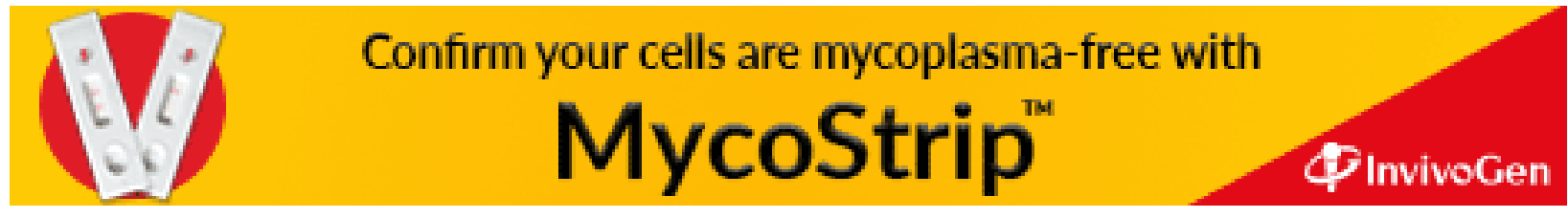

